\title{
Factores Interpersonales que influyen en el embarazo en adolescentes
}

\section{Interpersonal factors that influence adolescent pregnancy}

\author{
Margarita Lazcano-Ortiz ${ }^{a}$, Victoria A. Castillo-Ramírez ${ }^{b}$, Alejandra Díaz-Hernández ${ }^{b}$, Ana \\ K. Martiniano-María ${ }^{b}$, Arely Rodríguez- Mena ${ }^{b}$, Yuridia Vargas- Valencia ${ }^{b}$, Abigail \\ Villanueva-Estrada ${ }^{b}$, Reyna C. Jiménez-Sánchez ${ }^{c}$, María L. Sánchez-Padilla ${ }^{c}$
}

\begin{abstract}
:
Introduction: Adolescent pregnancy is a social problem that has increased in recent years due to factors that influence, some of these are lack of information about sexuality and deficiencies in the use of contraceptive methods. Objective: Determine interpersonal factors that influence adolescent pregnancy. Material and method: Correlational, transversal and descriptive study, not experimental. Inclusion criteria: pregnant teenagers from 12 to 19 years old. Non-probabilistic sampling for convenience, shows: 31 pregnant teenagers from the Dr. Jesús Corona del Rosal de Pachuca Health Center. The instruments were used; The first, prepared by the authors, measures "Interpersonal factors that influence pregnancy"; validated, 27 items of multiple choice, the second PSS-Fa (Perceived social support from family) of the authors: González Ramírez, R. Landero Hernández (2012), Cronbach's alpha 0.758. Results: the average age was 16 years $(\mathrm{SD}=.703: 16-18) .77 \%$ are in free union, $52 \%$ are engaged in household activities. $65 \%$ have access to popular insurance, $94 \%$ consider that the relationship with their partner is stable. $68 \%$ began to consume alcohol at 16 years old and only $1 \%$ did so during their pregnancy. $64 \%$ do not know the meaning of sex education. $84 \%$ do not know that it is a contraceptive method. $83 \%$ report feeling supported by their family. Conclusion: According to the data obtained, the interpersonal factor that influences adolescent pregnancy is the lack of knowledge about sex.
\end{abstract}

Keywords:

Pregnancy, adolescence, interpersonal factors

Resumen:

Introducción: El embarazo adolescente es un problema social que ha aumentado en los últimos años por factores tales como falta de información sobre sexualidad y deficiencias en el uso de los métodos anticonceptivos. Objetivo: Determinar factores interpersonales que influyen en el embarazo adolescente. Material y método: Estudio correlacional, transversal y descriptivo, no experimental. Una muestra de 31 adolescentes embarazadas del Centro de Salud Dr. Jesús Corona del Rosal de Pachuca Muestreo no probabilístico por conveniencia. Se utilizaron dos instrumentos; el primero elaborado por las autoras, mide "Factores interpersonales que influyen en el embarazo"; validado, con 27 ítems de opción múltiple, el segundo PSS-Fa (Perceived social support from family) de los autores: González Ramírez, R. Landero Hernández (2012) con un Alfa de Cronbach 0.758. Resultados: el promedio de edad fue de 16 años (DE = .703:16 - 18). El 77\% está en unión libre, el 52\% se dedica a las actividades del hogar. El $65 \%$ tiene acceso al seguro popular, el $94 \%$ considera que la relación con su pareja es estable. El $68 \%$ inicio a consumir alcohol a los 16 años y solo el $1 \%$ lo hizo durante su embarazo. El 64\% no sabe el significado de educación sexual. El 84\% desconoce que es un método anticonceptivo. El 83\% refiere sentirse apoyada por su familia. Conclusión: De acuerdo con los datos obtenidos, el factor interpersonal que influye en el embarazo adolescente es la falta de conocimientos sobre temas de educación sexual.

Palabras Clave:

Embarazo, adolescencia, factores interpersonales

${ }^{a}$ Autor de Correspondencia, Profesor Investigador, SNI, Universidad Autónoma del Estado de Hidalgo, Instituto de Ciencias de la Salud, Área académica de Enfermería. https://orcid.org/0000-0002-9653-9291. Pachuca Hgo. México, Email: margarita_lazcano@uaeh.edu.mx

Alumnos de Licenciatura en Enfermería de la Universidad Autónoma del Estado de Hidalgo, Instituto de Ciencias de la Salud, Área académica de Enfermería, Pachuca Hgo. México.

${ }^{c}$ Profesor investigador Universidad Autónoma del Estado de Hidalgo, Instituto de Ciencias de la Salud, Área académica de Enfermería, 


\section{Introducción}

El embarazo adolescente es un problema social que ha ido en aumento en los últimos años debido a diversos factores que influyen para que se presente en la sociedad. ${ }^{[1]}$ Algunos de estos factores son falta de información objetiva sobre sexualidad, deficiencias en el uso y manejo de los diferentes métodos anticonceptivos existentes, inicio de la vida sexual a edades cada vez menores, adicciones, problemas familiares, entre otros. ${ }^{[2]}$

Los puntos mencionados anteriormente son importantes para que las y los adolescentes cuenten con una orientación sexual adecuada, y que, a su vez, llegue a cubrir todas sus dudas e inquietudes respecto a su sexualidad y a la forma de ejercerla sin riesgo. La implementación de talleres, programas y políticas conducentes es realmente de mucha importancia; incrementar conocimientos sobre los diferentes métodos anticonceptivos a los que pueden recurrir, el uso correcto de los mismos, así como de las ventajas y desventajas de cada uno de los métodos. ${ }^{[1]}$

Las consecuencias del embarazo en los adolescentes son diversas y pueden clasificarse principalmente en físicas, psicológicas y sociales. ${ }^{[3]}$ Algunos de los efectos que pueden manifestarse son: interrupción del embarazo, trastornos emocionales como la depresión y la frustración, deserción escolar, abandono del hogar, limitaciones laborales, entre otros. ${ }^{[4]}$

A nivel mundial se ha visto el incremento de embarazo en adolescentes donde la Organización Mundial de la Salud ${ }^{[3]}$ ha señalado que anualmente alrededor de 16 millones de niñas entre 15 y 19 años son madres. En las Estadísticas Sanitarias Mundiales 2014 se indica que la tasa media de natalidad mundial entre las adolescentes de 15 a 19 años es de $49 \%$.

A nivel nacional, el embarazo adolescente es considerado un problema de Salud Pública grave ya que cada año nacen aproximadamente 40.355 recién nacidos vivos hijos de madres adolescentes y 1.175 hijos de madres menores de 15 años. Así, la incidencia anual del embarazo adolescente en nuestro país es de $16,16 \%$ según el INEGI en el año $2014 .{ }^{[4]}$

En el desarrollo de este proyecto se abordaran distintos conceptos los cuales se relacionan a los aspectos interpersonales tomando en cuenta la importancia de cada uno; la adolescencia y de los cambios que ocurren en esta etapa, tanto en el hombre como en la mujer, haciendo mayor énfasis en el área emocional con la finalidad de comprender algunos de los aspectos por los cuales atraviesan los adolescentes en este periodo, se analiza la vida social en el adolescente, tanto las relaciones familiares, convivencia entre amigos y de la relación de pareja, conocida como noviazgo ${ }^{[5]}$

\section{Material y método}

Estudio correlacional, transversal y descriptivo. Diseño no experimental. Tipo de muestreo no probabilístico por conveniencia, con una muestra de 31 adolescentes embarazadas del Centro de Salud Dr. Jesús Corona del Rosal de Pachuca de Soto Hidalgo. Se utilizaron dos instrumentos de investigación; el primero elaborado por los autores para medir "Factores interpersonales que influyen en el embarazo" que fue validado, consta de 27 ítems de opción múltiple, el segundo fue una escala PSS-Fa (Perceived social support from family) de los autores: González Ramírez, R. Landero Hernández (2012), que mide la relación con la familia, consta de 16 ítems. Con una confiabilidad alfa de Cronbach de 0.758 .

\section{Resultados}

El promedio de edad fue de 16 años ( $D E=.703: 16-18$ ). Con respecto al estado civil el $77 \%$ de la muestra es unión libre. En cuanto a ocupación el $52 \%$ de la muestra se dedica a las actividades del hogar. Respecto a los servicios de salud con el que cuentan el $65 \%$ de la muestra tiene acceso al seguro popular. El $94 \%$ de la muestra considera que la relación con su pareja es 
estable. Con respecto al consumo de alcohol el 68\% inicio a consumirlo a los 16 años y solo el $1 \%$ de la muestra lo hizo durante su embarazo. En relación con los conocimientos acerca del término educación sexual el $64 \%$ de la muestra no sabe a qué se refiere ese término, y el $99 \%$ de la muestra refiere haber recibido información en la escuela. El $84 \%$ de la muestra desconoce que es un método anticonceptivo. Con respecto a la relación con su familia el $83 \%$ de la muestra refiere sentirse apoyada por su familia, y el $48 \%$ de la muestra refiere que a su familia le gusta escuchar lo que piensa.

Figura 1 muestra que casi todas (94\%) las adolescentes embarazadas estudiadas consideran que su relación es estable.

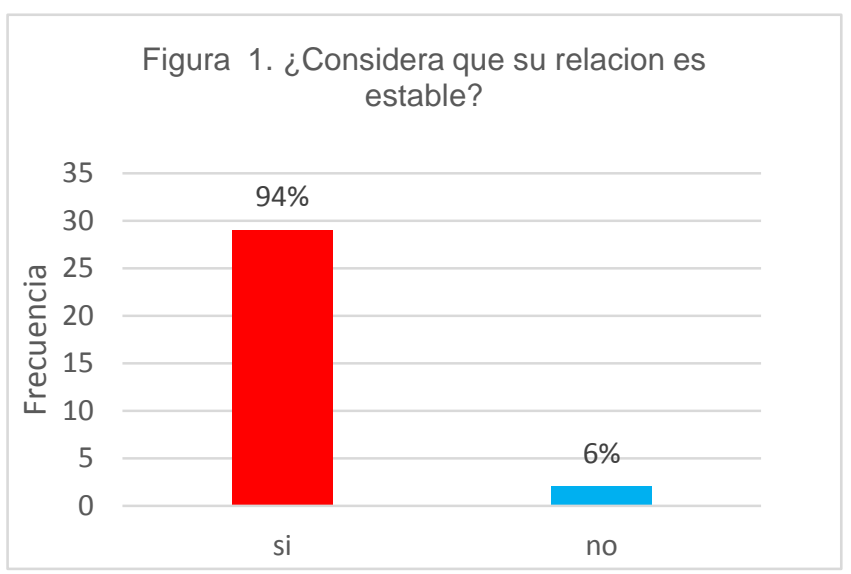

Figura 2 muestra que casi todas (98\%) las adolescentes embarazadas considera importante recibir educación sexual a temprana edad.

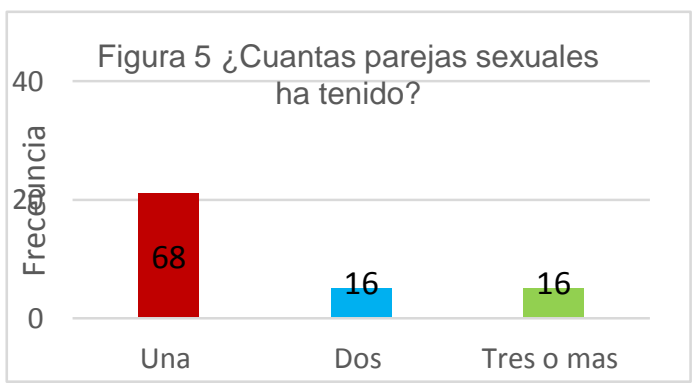

Figura 3 muestra que más de la mitad (68\%) de las adolescentes embarazadas encuestadas iniciaron a consumir alcohol a la edad de 16 años.

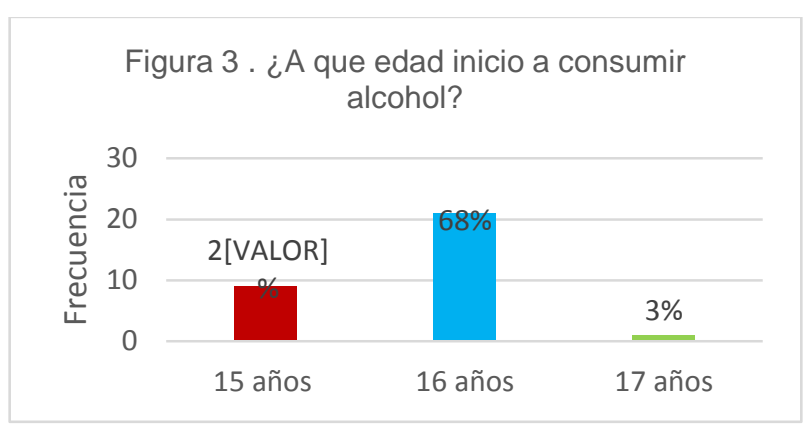

Figura 4 muestra que más de la mitad (68\%) de las mujeres embarazadas encuestadas refiere que inicio su vida sexual activa a la edad de 16 años

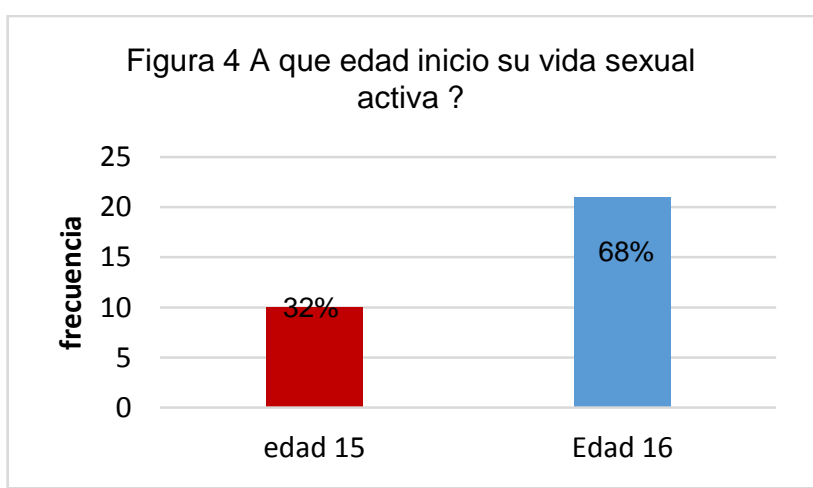

La figura 5 muestra que más de la mitad (68\%) de las mujeres embarazadas refiere que solo tuvo una pareja sexual.

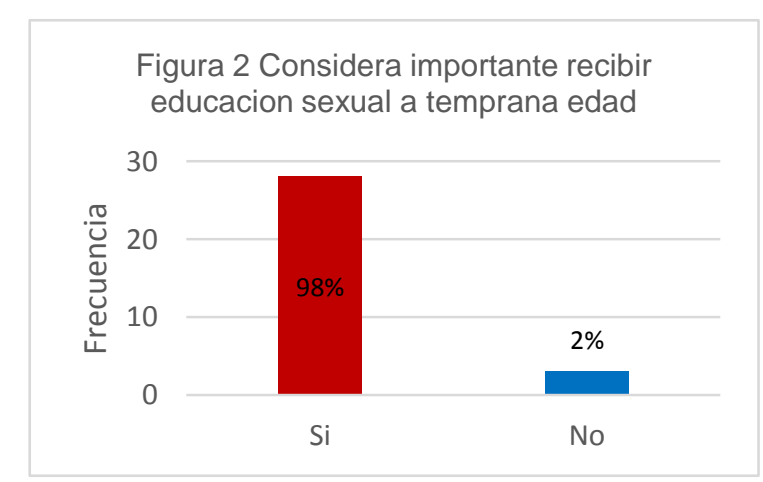




\section{Discusión}

El embarazo adolescente es un problema social que ha ido en aumento en los últimos años debido a diversos factores que influyen para que este suceso se presente en la sociedad. Algunos de estos factores son falta de información objetiva sobre sexualidad, deficiencias en el uso y manejo de los diferentes métodos anticonceptivos existentes, inicio de la vida sexual a edades cada vez menores, adicciones y problemas familiares, de ahí la importancia de prevenir el embarazo adolescente e indagar que lo predispone a una edad temprana; el objetivo de este estudio es determinar los factores interpersonales asociados en el embarazo en adolescentes por lo que se encuestaron a 31 adolescentes embarazadas en el centro de salud de Pachuca, "Jesús Corona del Rosal" obteniendo los resultados antes mencionados.

En el estudio que realiza Alarcón. $R,{ }^{[6]}$ "Factores que influyen en el embarazo en la adolescencia" encuentra que las personas tienen una relación adecuada con sus padres, es decir se sienten apoyadas; se observa similitud con este estudio donde las mujeres refieren tener una buena relación con su familia, y esta es la que les da ánimo. Según Coloma la familia es el primer elemento socializador en cuyo seno se educa y forma al individuo, en ella se aprenden las nociones básicas para vivir en sociedad, se produce un determinado sistema de valores, normas y creencias que le sirven al adolescente para construir el concepto de sí mismo y alcanzar una identidad personal. ${ }^{[7]}$

Con respecto a la educación sexual, Márquez ${ }^{[8]}$ refiere en su tesina "Factores que influyen en un embarazo en adolescentes y sus implicaciones en las esferas psicosociales" que uno de los factores que originan el problema de embarazos en las adolescentes es la información deficiente respecto a la sexualidad en los adolescentes; se encuentra similitud con en este en donde las mujeres refieren que no saben que es la educación sexual. Con lo anterior es necesario que los padres orienten a sus hijos desde pequeños y sobre todo dentro del hogar respecto a la educación sexual, esto es que desde pequeños no comiencen a originar en sus hijos tabúes en cuanto a la sexualidad.

En el estudio realizado por Peña J. [9] "Consumo de alcohol en mujeres embarazadas", se encuentra que el consumo del alcohol durante el embarazo es mínimo, existe una relación con el estudio "Factores interpersonales que influyen en el embarazo en adolescentes en el Centro de Salud ,Pachuca” puesto que el porcentaje de las mujeres que consumieron alcohol durante el embarazo también es mínimo, esto quiere decir que tienen conocimientos acerca de las consecuencias que ocasiona durante la etapa gestacional.

Coincide con este estudio la investigación de Peña ${ }^{[9]}$ "Consumo de alcohol en mujeres embarazadas" quien también encuentra que el consumo de alcohol durante el embarazo adolescente es mínimo, esto podría explicarse por la existencia de una tendencia natural a dejar de consumir alcohol durante el embarazo reportada por Jacobson, ${ }^{[10]}$ que alcanza hasta $80 \%$ según el estudio realizado en mujeres de bajos ingresos, pero vuelve a su nivel habitual pre-embarazo después del parto.

\section{Conclusión}

Se puede concluir que el factor interpersonal que influye en el embarazo adolescente es la falta de conocimientos en temas de educación sexual. El índice de embarazos sigue en aumento y las edades con mayor prevalencia según el estudio realizado son adolescentes de 16 años de edad, cifras que son alarmantes para la sociedad. La mayoría de estas adolescentes viven con su pareja, manteniendo una relación estable y fomentando el respeto; ya no estudian, solo se dedican a las actividades del hogar, cuentan con seguro popular como servicio de salud. En temas de planificación familiar aún no saben identificar que es un método anticonceptivo, solo habían escuchado del condón y es el método que 
más han utilizado pero no les dio resultado porque no supieron como emplearlo.

El consumo de alcohol no fue un factor que influya en el desarrollo de embarazos en adolescentes, se encontró que solo una cuarta parte lo consumió y solo el $1 \%$ lo hizo durante su embarazo, porque de acuerdo con los resultados la mayoría de las adolescentes esta consiente de los riesgos que causa ingerir esta sustancia durante el embarazo.

Otro factor fue la promiscuidad, pero sobre ese tema no se encontró nada relevante, la mayoría de las adolescentes mantuvieron relaciones sexuales solo con su pareja actual.

Otro factor que se investigo fue el apoyo de la familia y esto tampoco influye de manera directa ya que la mayoría de los adolescentes lleva una relación estable con su familia.

\section{Referencias}

[1] Ángeles, L. M. Embarazo Adolescente y madres jóvenes en México: Una visión desde el Promajoven. [en línea]. 2015 [citado 20 de octubre de 2018]; Recuperado en: https://www.promajoven.sep.gob.mx/files/materiales/Embarazo_Adolesce nte.pdf

[2] González, J. D. Prevención del embarazo adolescente. [en línea]. 2015 [citado 10 de octubre de 2018]; Recuperado en: https://www.google.com/search?q=González,+J.+D.+(28+de+12+de+201 5).+Prevención+del+embarazo+adolescente.+Grupo+Milenio,+págs.+223.\&tbm=isch\&source=univ\&sa

[3] OMS. Desarrollo en la adolescencia . [en línea]. [citado 8 de noviembre de 2018]; Recuperado en: https://www.who.int/maternal child adolescent/topics/adolescence/dev/es

[4] INEGI. Incidencia del embarazo. [en línea]. 2014. [citado 19 de noviembre de 2018]; Recuperado en: https://www.inegi.org.mx/

[5]Vázquez, G. R. salud y sexualidad [en línea]. 2012 . [citado 22 de noviembre de 2018]; Recuperado en: http://www.msal.gob.ar/saludsexual/downloads/aqq.pdf

[6]Alarcón, A. R. . Factores que influyen en el embarazo en la adolescencia. Revista Cubana de Enfermeria. . [en línea]. 2009 . [citado 8 de diciembre de 2018]; Recuperado en:

http://scielo.sld.cu/scielo.php?script=sci_arttext\&pid=S086403192009000100007

[7] Savater F. El valor de educar. Redalcyc [en línea]. 1997 [citado 22 de enero de 2019]; (9): 283-287

Recuperado en: http://www.redalyc.org/pdf/267/26700915.pdf

[8] Márquez, S. MG. Cardoza Morales P (dir). Factores que influyen en el embarazo adolescente y sus implicaciones en las esferas psicosociales . [tesis licenciatura en Internet ], [Mexico]: Universidad Pedagogica Nacional; 2015 [citado 12 enero 2019]: Recuperado en: http://200.23.113.51/pdf/25184.pdf
[9] Peña, J, Matute E.Consumo de alcohol en mujeres embarazadas. Sociedad. 2010, 15(47):211-229.

Recuperado

en:

http://www.scielo.org.mx/pdf/espiral/v16n47/v16n47a7.pdf

[10] Castillo Diaz R. Alonso Castillo MM (dir) Gomez Meza MV (dir). Creencias y consumo de alcohol en mujeres embarazadas. [tesis maestria en Internet ]. [Mexico]: Universidad Autònoma de Nuevo Leòn, 2005 [citado 22 enero 2019]: Recuperado en http://eprints.uanl.mx/5563/1/1020150988. 